A 76-year-old man with a one-month history of painful swelling in the left lower leg was admitted to our department. He had received treatment for CML with $20 \mathrm{mg} /$ day dasatinib for the previous three years. He had a history of cerebral infarction and Stanford type A aortic dissection. Examination revealed that the lower portion of his left leg exhibited dark red erythema, oedema, and was warm to touch (figure 1A). He was febrile, and his body temperature was $37.5^{\circ} \mathrm{C}$. Laboratory tests revealed a normal white blood cell count, but the level of $\mathrm{C}$-reactive protein was elevated (4.82; normal range $<0.30 \mathrm{mg} / \mathrm{dL}$ ). Bacterial cellulitis was initially suspected based on this presentation. Treatment with bed rest, leg elevation, and antibiotics (intravenous ampicillin/sulbactam) was initiated, however, the skin lesions gradually spread peripherally. Further examination with computed tomography angiography and ultrasound revealed no evidence of deep venous thrombosis or venous stasis. Histopathological examination revealed numerous inflammatory cells in the subcutaneous fat lobules and septa (figure 1B), which comprised lymphocytes, eosinophils, and histiocytes (figure 1C). Tissue cultures for bacteria, acid-fast bacilli, and fungi were all negative. From these findings, it was suspected that the patient had drug-induced panniculitis, and subsequently, treatment with dasatinib was discontinued. The skin lesions gradually disappeared after one week following the discontinuation of dasatinib treatment (figure 1D). The discontinuation of dasatinib therapy did not exacerbate the patient's CML, and the skin lesions did not recur thereafter.

Dasatinib-induced panniculitis has been reported in only two patients to date, however, the clinicopathological features differed from those of the case presented herein [3]. TKI-associated panniculitis is a rare adverse event characterized by multiple erythematous nodules on the arms and legs $[4,5]$. In contrast, this case presented with diffuse dark red erythema and oedema on the leg mimicking cellulitis. Although TKIs exert their antitumour effects through inhibition of the aberrant kinase activity of the BCR-ABL1 fusion protein, produced by the Philadelphia chromosome translocation, the exact pathomechanism associated with drug-induced panniculitis is unclear. Panniculitis has been reported to occur several months or even up to one year after initial treatment with TKIs [4]. Our case highlights that disease can present even after years of treatment. TKI-induced panniculitis is histopathologically characterized by acute inflammatory infiltrates, primarily comprising neutrophils within the lobules [3,4]. In this case, however, lymphocytic and eosinophilic infiltrates also extended into the septa, which may reflect long-term or severe panniculitis. TKIinduced panniculitis has previously been reported to rapidly improve following withdrawal of the causative drug, as in our case. The reintroduction of TKI treatment may be possible in combination with systemic corticosteroids [4]. Although drug-induced panniculitis is rare, dermatologists should be aware that it is a potential adverse event during dasatinib treatment. Additional evidence is necessary, which will aid in our understanding of the pathomechanism and clinicopathological features of TKI-associated panniculitis.

Disclosure. Financial support: none. Conflicts of interest: none.
${ }^{1}$ Department of Dermatology, Asahikawa Medical University, Asahikawa, Japan

${ }^{2}$ Department of Diagnostic Pathology, Asahikawa Medical University, Asahikawa, Japan <iinuma@asahikawa-med.ac.jp> inhibitors in chronic myeloid leukaemia: which, when, for whom? Nat Rev Clin Oncol 2017; 14: 141-54.

2. Delgado L, Giraudier S, Ortonne N, et al. Adverse cutaneous reactions to the new second-generation tyrosine kinase inhibitors (dasatinib, nilotinib) in chronic myeloid leukemia. J Am Acad Dermatol 2013; 69: 839-40.

3. Assouline S, Laneuville P, Gambacorti-Passerini C. Panniculitis during dasatinib therapy for imatinib-resistant chronic myelogenous leukemia. N Engl J Med 2006; 354: 2623-4.

4. Zhang $M$, Hassan KM, Musiek A, Rosman IS. Ponatinib-induced neutrophilic panniculitis. J Cutan Pathol 2014; 41:597-601.

5. Kitayama N, Otsuka A, Hamamoto C, et al. Nilotinib-induced panniculitis in a patient with chronic myelogenous leukaemia. J Eur Acad Dermatol 2017; 31: e418-9.

doi:10.1684/ejd.2020.3946

\section{A case of a gibberellin-regulated protein- positive patient allergic to various fruits}

Gibberellin-regulated protein (GRP) was recently identified as a new allergen in fruit allergies [1]. GRP is present in a wide range of fruits and causes allergic symptoms, including anaphylaxis. A 17-year-old woman visited our department because of a reddish rash on her trunk, a feeling of discomfort in the oral cavity, and difficulty breathing immediately after she ate a Citrus unshiu and then exercised. Her medical history included pollinosis, oral discomfort after consuming a peach at 10 years old, an itching sensation in the oral cavity after eating lemon cake following exercise at 16 years old, and discomfort in the oral cavity after drinking orange juice and eating a grapefruit at 17 years old. In addition, she reported a sense of discomfort in the oral cavity immediately after ingesting a Japanese apricot (ume).

According to the ImmunoCAP® (Thermo Fisher Scientific, Uppsala, Sweden) test, specific IgE was positive for cedar pollen $(47.3 \mathrm{UA} / \mathrm{mL})$, cypress pollen $(12.1 \mathrm{UA} / \mathrm{mL})$, peach $(1.68 \mathrm{UA} / \mathrm{mL})$ and orange $(0.37 \mathrm{UA} / \mathrm{mL})$. The serum $\mathrm{IgE}$ levels (ImmunoCAP®; Thermo Fisher Scientific) for the peach components rPru p 1 (Bet v 1-homolog), rPru p 3 (lipid transfer protein) and rPru p 4 (profilin) were negative. IgE specific for native peach GRP (nPru p 7) tested positive based on an enzyme-linked immunosorbent assay (13.2 U/mL). A skin prick test (SPT) was performed using the prick-prick method (see supplementary material). The patient showed a positive reaction to both non-heated and heated orange pulp, non-heated orange pericarp, both nonheated and heated $C$. unshiu pulp, heated $C$. unshiu pericarp, heated grapefruit pulp and pericarp, both non-heated and heated lemon pulp, non-heated lemon pericarp, both nonheated and heated peach pulp and pericarp, canned peach, 


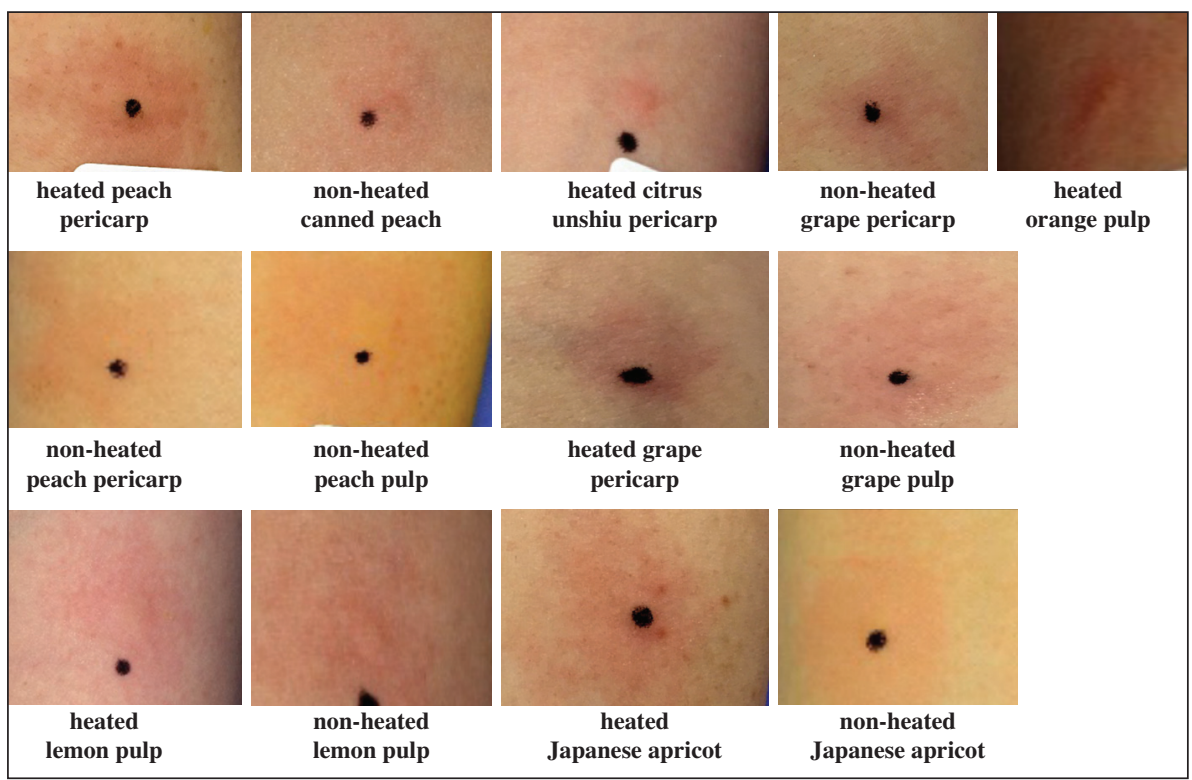

Figure 1. Examples of positive reactions based on skin prick testing.

and both non-heated and heated Japanese apricot (figure 1). One year after the examination, discomfort in the oral cavity was reported immediately after ingesting grapes. The patient showed a positive reaction to both non-heated and heated grape pulp and pericarp based on a SPT (figure 1). GRP was identified as the fifth peach allergen, Pru $p$ 7 (peamaclein) [1]. This is registered under the WHOInternational Union of Immunological Societies (IUIS) as follows: peach Pru p 7, orange Cit s 7, Japanese apricot Pru m 7, pomegranate Pun g 7 and cypress pollen Cup s 7 [25]. Cross-reactivity between four fruit-derived GRPs, Pru p 7, Pru m 7, Cit s 7 and Pun g 7, has been proven [2-4]. Tuppo et al. reported important insight into potential crossreactivity between fruit-derived and pollen-derived GRPs [5]. In the present case, IgE specific to cypress pollen and Pru p 7 tested positive. We did not perform a suppression test to confirm cross-antigenicity, as this may have caused a cross-reaction with these fruit GRPs, as a sensitizing antigen for cypress pollen. GRP is resistant to heat and digestive enzymes [1], therefore allergic symptoms can appear even when GRP-containing food is heated. In the present case, symptoms occurred after ingesting a variety of fruits. Inomata et al. reported that for patients mono-sensitized to orange GRP, multiple fruits were causative [3]. Peach is the most common; others include Japanese apricot, grape, cherry, lemon, grapefruit, etc. [3]. We advised the patient to refrain from eating non-heated/heated peaches, citrus fruits, Japanese apricots and grapes as well as processed products containing any of these fruits. Based on an analysis to determine homology, Pru p 7 was shown to be similar to Pun g 7 (90\% identity) [4]. Unfortunately, we were unable to obtain pomegranate, and so could not perform a SPT for this fruit. We advised the patient to refrain from consuming pomegranate, as there was a possibility that she might develop allergic symptoms in response.

GRP has been found to be present in many plants, and we continue to monitor the patient carefully.

Disclosure. Conflicts of interest: none.
Open Access. This article is licensed under a Creative Commons Attribution 4.0 International License, which permits use, sharing, adaptation, distribution and reproduction in any medium or format, as long as you give appropriate credit to the original author(s) and the source, provide a link to the Creative Commons licence, and indicate if changes were made. The images or other third party material in this article are included in the article's Creative Commons licence, unless indicated otherwise in a credit line to the material. If material is not included in the article's Creative Commons licence and your intended use is not permitted by statutory regulation or exceeds the permitted use, you will need to obtain permission directly from the copyright holder. To view a copy of this licence, visit http://creativecommons.org/licenses/by/4.0/.

Supplementary data. Supplementary data associated with this article can be found, in the online version, at doi:10.1684/ejd.2020.3949.

Table S1: Results based on skin prick tests. A skin prick test was performed using the prick-prick method and considered positive when the average wheal diameter induced by the allergen was $50 \%$ larger than the positive control response induced by histamine chloride at $10 \mathrm{mg} / \mathrm{mL}$. The size of the wheal and erythema were measured after 15 minutes later. We heated various fruits in a microwave oven at $500 \mathrm{~W}$ for 2 minutes. +: positive; -: negative; ND: not done.

${ }^{1}$ Department of Dermatology, Iwate
Medical University School of
Medicine, Japan
${ }^{2}$ Department of Environmental,
Immuno-Dermatology, Yokohama
City University Graduate School of
Medicine, Yokohama, Japan
<mnakagaw@ iwate-med.ac.jp>
<hamano@iwate-med.ac.jp>

Michiyo NAKAGAWA ${ }^{1}$ Miho HANADA ${ }^{1}$ Naoko INOMATA ${ }^{2}$ Hiroo AMANO ${ }^{1}$

(C) The Author(s) 2021 
1. Inomata N, Okazaki F, Moriyama T, et al. Identification of peamaclein as a marker allergen related to systemic reactions in peach allergy. Ann Allergy Asthma Immunol 2014; 1 12: 175-7.

2. Inomata N, Miyakawa $M$, Aihara M. Gibberellin-regulated protein in Japanese apricot is an allergen cross-reactive to Pru p 7. Immun Inflamm Dis 2017; 5: 469-79.

3. Inomata N, Miyakawa M, Ikeda N, Oda K, Aihara M. Identification of gibberellin-regulated protein as a new allergen in orange allergy. Clin Exp Allergy 2018; 10: 1-12.

4. Tuppo L, Alessandri C, Pasquariello MS, et al. Pomegranate cultivars: identification of the new lgE- binding protein pommaclein and analysis of antioxidant variability. J Agric Food Chem 2017;65: 2702-10.

5. Tuppo L, Alessandri C, Giangrieco I, et al. Isolation of cypress gibberellin-regulated protein: analysis of its structural features and lgE binding competition with homologous allergens. Mol Immunol 2019; 114:189-95

doi:10.1684/ejd.2020.3949

\section{Erythro-papular type drug eruption caused by fosravuconazole L-lysine ethanolate}

Fosravuconazole L-lysine ethanolate (F-RVCZ), recently released in Japan, is an azole-based antifungal medicine used for treating onychomycosis. We report a case of erythro-papular drug eruption caused by F-RVCZ.

A 66-year-old man underwent vitreous body surgery for a cataract in July 2019. He started systemic treatment with cefaclor on the day of the operation, and oedematous erythema appeared on his body the following day. Cefaclor was thought to have caused the drug eruption and he was switched to levofloxacin. Subsequently, the erythema spread to his whole body, and he was re-examined three days after the first examination.

Oedematous erythema without itchiness was present mainly on his torso and lower limbs, and palpable erythema were scattered on the patient's trunk (figure $1 A$ ). He did not present with fever. His blood test results were as follows: white blood cells at $8300 / \mu \mathrm{L}$ (4\% eosinophils, $83 \%$ neutrophils, $8 \%$ lymphocytes, $5 \%$ monocytes), C-reactive protein at $3.71 \mathrm{mg} / \mathrm{dL}$, aspartate aminotransferase at $15 \mathrm{U} / \mathrm{L}$, and alanine aminotransferase at $21 \mathrm{U} / \mathrm{L}$. Histopathologically, liquefaction was observed, but necrotic keratinocytes were not detected in the skin lesion. There was lymphocyte permeation around the blood vessels of the dermis, and eosinophils were visible (figure $1 B, C$ ). He was treated with prednisolone at $30 \mathrm{mg} /$ day for the prolonged druginduced skin eruption, and his symptoms initially improved, but the oedematous erythema eventually resumed spreading. He had experienced a drug-induced skin eruption with aztreonam in the past and had a history of related hospitalisation. The patient's medical history also included the use of telmisartan for hypertension, F-RVCZ for onychomycosis, and mecobalamin tablets (methycobal) for spinal stenosis. The patient had been taking telmisartan and methycobal for several years but had only started using F-RVCZ for 41 days. F-RVCZ treatment was stopped, and the erythema resolved with continued treatment with prednisolone at $30 \mathrm{mg} /$ day. Prednisolone administration was discontinued after approximately one month of treatment. The onychomycosis was treated with efinaconazole solution after stopping oral F-RVCZ. Lymphocyte transformation test (LTT) was conducted for cefaclor and levofloxacin on Day 6 after the drug eruption occurred, and for F-RVCZ on Day 20. The stimulation indices for cefaclor, levofloxacin, and F-RVCZ were $97 \%, 108 \%$, and $189 \%$, respectively, as per the LTT test results, and the patient was diagnosed with fosravuconazole-induced erythro-papular-type drug eruption. LTT for itraconazole, another azole-based antifungal medicine, was not conducted. Mecobalamin tablets and telmisartan were continued thereafter, and no recurrence of erythema was observed.

Ravuconazole (RVCZ) is a fourth-generation azole-based antifungal drug with strong antimycotic activity, but it may cause adverse drug interactions or hepatic dysfunction. F-RVCZ is a precursor of RVCZ; when F-RVCZ is absorbed in the body, it is hydrolysed immediately and converted to RVCZ, which is the active ingredient. Because the pharmacokinetics of F-RVCZ are improved after its transformation to the prodrug, it has high transitivity to the nails. Itraconazole and terbinafine are oral therapeutic drugs generally used for onychomycosis in Japan. Since F-RVCZ can interact with other drugs, the risk of severe liver damage is a matter of concern. Drugs that show pharmacological interactions with F-RVCZ have been reported to be mainly limited to those metabolised by cytochrome P450 3A; therefore, there are not many concomitant drugs that require attention. In the Phase III clinical trials of FRVCZ, no serious liver damage was reported, and abnormal blood results and gastrointestinal problems were the main issues [1].

To our knowledge, this is the first report on drug eruption caused by F-RVCZ. F-RVCZ is frequently used for treating onychomycosis in Japan; therefore, dermatologists and clinicians must be aware that F-RVCZ might induce eruptions on the skin.

Disclosure. Financial support: none. Conflicts of interest: none.
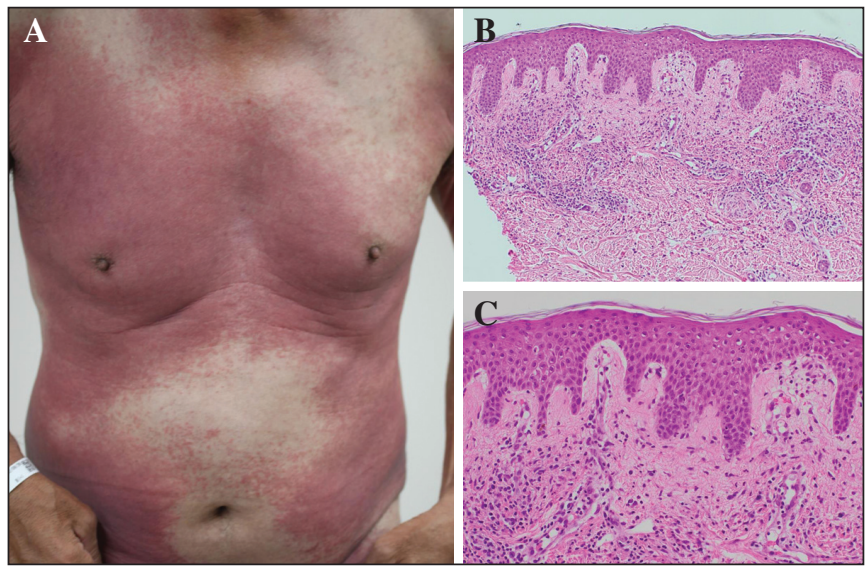

Figure 1. Clinical features and pathological findings of the patient. A) Erythema scattered on the torso. B, C) Histopathological examination showing erythema with perivascular inflammation in the upper dermis (B), and liquefaction in the junction between the epidermis and dermis, with lymphocytes and eosinophils visible around the blood vessels of the dermis $(\mathbf{C})$. 